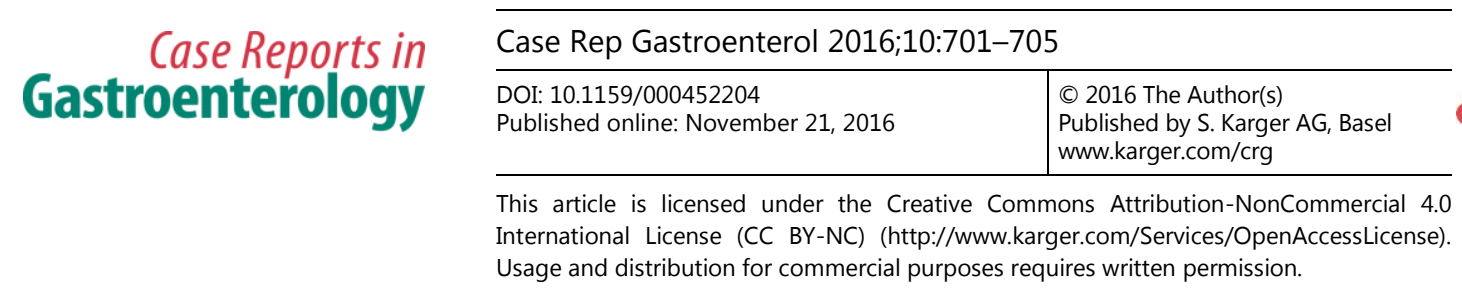

\title{
Hepatic Encephalopathy due to Congenital Multiple Intrahepatic Portosystemic Venous Shunts Successfully Treated by Percutaneous Transhepatic Obliteration
}

\author{
Shinsuke Takenaga $^{a} \quad K$ Kenichi Narita ${ }^{b}$ Yo Matsui ${ }^{c}$ Kunihiko Fukuda $^{b}$ \\ ${ }^{a}$ Department of Radiology, The Jikei University Katsushika Medical Center, Tokyo, Japan; \\ ${ }^{b}$ Department of Radiology, The Jikei University School of Medicine, Tokyo, Japan; \\ 'Department of Radiology, Fuji City General Hospital, Fuji, Japan
}

\section{Keywords}

Intrahepatic portosystemic venous shunt $\cdot$ Hepatic encephalopathy $\cdot$ Embolization

\begin{abstract}
Hepatic encephalopathy due to intrahepatic portosystemic venous shunts (IPSVS) in a noncirrhotic condition is rare. Here we report a rare case of a patient with congenital multiple IPSVS successfully treated by percutaneous transhepatic obliteration. The patient was a 67year-old woman who presented to our hospital with progressive episodes of consciousness disorder and vomiting. Laboratory tests revealed hyperammonemia $(192.0 \mu \mathrm{g} / \mathrm{dL})$, and computed tomography revealed multiple IPSVS in both lobes. There was no evidence of underlying liver disease or hepatic trauma. Transcatheter embolization for IPSVS was performed because conservative therapy was not sufficiently effective. After endovascular shunt closure, hepatic encephalopathy improved. The serum ammonia level normalized during the 5-year follow-up period. Thus, transcatheter embolization may be an effective therapy for patients with symptomatic and refractory IPSVS. Careful follow-up is necessary for portal hypertension-related complications after transcatheter embolization for IPSVS.




\section{Introduction}

Hepatic encephalopathy due to intrahepatic portosystemic venous shunts (IPSVS) without portal hypertension is a rare condition. IPSVS is a vascular anomaly, which is defined as a communication between the portal and systemic venous circulations, measuring more than $1 \mathrm{~mm}$ in diameter and being at least partially located inside the liver [1].

Interventional treatment for IPSVS is required if the conservative treatments (restriction of protein and administration of nonabsorbable antibiotics and lactulose) for hepatic encephalopathy are not sufficiently effective. Because of the invasiveness of surgical interventions, such as shunt ligation, hepatic resection, or liver transplantation, transcatheter embolization of the causative vessel has been widely used for the last 3 decades [1-9].

To our knowledge, only 2 case reports $[3,5]$ in which multiple IPSVS in both hepatic lobes with hepatic encephalopathy were treated by transcatheter embolization have been published in English-language literature. In addition, in both of these cases, transileocolic obliteration was performed through a small abdominal incision, with the patient under general anesthesia.

Here, we report a case diagnosed with hepatic encephalopathy due to congenital multiple IPSVS in both hepatic lobes, which were successfully treated by percutaneous transhepatic obliteration.

\section{Case Presentation}

A 67-year-old woman presented with orientation disturbance, bradykinesia, and vomiting for 2 weeks at another hospital, where she had undergone rehabilitation for cerebellar infarction. Brain computed tomography (CT) in the hospital revealed no findings that could account for the symptoms. Because of a gradual progression in her symptoms, she was transferred to our hospital.

On admission, she was normotensive and afebrile without dyspnea. Physical examination revealed a mild gait ataxia due to cerebellar infarction. Flapping tremor, abdominal tenderness, hepatosplenomegaly, edema, and ascites were absent. Laboratory tests revealed hyperammonemia $(192.0 \mu \mathrm{g} / \mathrm{dL})$. Other laboratory tests related to liver function revealed alanine aminotransferase $14 \mathrm{IU} / \mathrm{L}$, aspartate aminotransferase $16 \mathrm{IU} / \mathrm{L}$, alkaline phosphatase $347 \mathrm{IU} / \mathrm{L}$, lactate dehydrogenase $273 \mathrm{IU} / \mathrm{L}$, cholinesterase $137 \mathrm{IU} / \mathrm{L}$, total bilirubin 1.4 $\mathrm{mg} / \mathrm{dL}$, albumin $2.7 \mathrm{~g} / \mathrm{dL}$, international normalized ratio of prothrombin time 1.60 (after oral administration of warfarin), and a platelet count of $126 \times 10^{3} / \mu \mathrm{L}$. There was no evidence of underlying liver diseases, such as hepatitis $B$, hepatitis $C$, and primary biliary cirrhosis. Furthermore, she had no history of alcohol consumption or liver injury. Enhanced CT images revealed multiple direct communications between portal and hepatic veins (Fig. 1). Thus, we diagnosed this patient with hepatic encephalopathy due to congenital multiple IPSVS.

In spite of conservative therapies, including low-protein diet, administration of lactulose, branched-chain amino acids, and kanamycin, and control of defecation, there was no sufficient reduction in the patient's serum ammonia levels or improvement in her symptoms. Therefore, endovascular shunt closure was performed.

After an intrahepatic portal vein branch was punctured with sonographic guidance under local anesthesia, a 6-Fr sheath introducer (S-one sheath, Terumo Clinical Supply, Gifu, Japan) was inserted into a right portal vein branch. Digital subtraction angiography from the 
main portal trunk showed multiple portosystemic venous shunts (Fig. 2a). The portal venous pressure was 10.0 (normal range, 5-10) mm Hg. A 5-Fr balloon catheter (Selecon MP II, Terumo Clinical Supply) was inserted into each portal vein branch, and a microcatheter (Progreat $\beta$, Terumo Clinical Supply) was coaxially advanced into the shunt point. Twentyone detachable coils (IDC, Boston Scientific, Watertown, MA, USA) and 42 pushable coils (Tornado coil, Cook, Bloomington, IN, USA) were deployed during balloon inflation. When ten shunt vessels were embolized, portal venous pressure was elevated to $18.1 \mathrm{~mm} \mathrm{Hg}$. Accordingly, we decided to complete the procedure, although there were a number of residual shunt vessels (Fig. 2b).

On the next day of the endovascular shunt closure, the serum ammonia level normalized. Although the patient continued the oral administration of lactulose, branched-chain amino acids, and kanamycin, neither hyperammonemia nor consciousness disorders occurred during the 5-year follow-up period after embolization (Fig. 3). In addition, upper gastrointestinal endoscopy showed no evidence of esophagogastric varices, which suggested a progression of portal hypertension.

\section{Discussion}

Although IPSVS usually occurs in patients who have liver cirrhosis or liver trauma, it rarely originates congenitally. This embryonal anomaly is thought to occur because of persistent anastomoses between cranial and caudal hepatic sinusoids, which comprise vitelline veins and the umbilical vein [10]. Most patients live asymptomatically while young. However, the incidence of hepatic encephalopathy increases with age because of a decreasing tolerance to ammonia in the aged brain [11]. In addition, the shunt ratio is also related to the development of hepatic encephalopathy. Park et al. [12] classified IPSVS into four types on the basis of anatomical variations in the shunt vessels of the liver: type 1, a single large vessel connecting the portal vein or its right branch to the inferior vena cava; type 2 , a localized peripheral shunt between the peripheral branches of the portal vein and hepatic veins in one hepatic segment; type 3, peripheral portal and hepatic veins connecting through an aneurysm; type 4, multiple communications between peripheral portal and hepatic veins diffusely distributed in both lobes. Due to the presence of multiple portosystemic venous shunts in both lobes, the case presented here corresponds to type 4, which is the rarest type.

Interventional treatment for IPSVS should be considered when patients are symptomatic and refractory to medications. Since being first reported by Ohtomo et al. [2], transcatheter embolization has been accepted as a noninvasive and effective treatment for symptomatic IPSVS [1-9]. The technical approaches of transcatheter embolization for IPSVS include transileocolic, percutaneous transhepatic, and retrograde transcaval embolization. Although transileocolic obliteration is feasible for the patients who have multiple shunts in both lobes, as in the present case, this procedure is invasive because a small abdominal incision under general anesthesia is required. We initially planned to perform the retrograde transcaval approach from the right internal jugular vein because of its noninvasive nature. However, it was difficult to advance the catheter into the shunt vessels, and antegrade portography could not be performed with this approach. Thus, we finally chose the percutaneous transhepatic approach.

In the present case, not all shunts were embolized because of the elevation of portal venous pressure. However, normal serum ammonia levels were maintained, and no episode of 
Takenaga et al.: Hepatic Encephalopathy due to Congenital Multiple IPSVS Successfully Treated by Percutaneous Transhepatic Obliteration

consciousness disorder occurred for 5 years after embolization. This suggests that the total quantity of shunt flow had significantly decreased.

Elevated portal venous pressure by a sudden blockage of shunt vessels may cause symptoms such as ascites and bleeding of esophagogastric varices. Hence, patient follow-up with upper gastrointestinal endoscopy and contrast-enhanced CT scan after embolization is necessary for detecting portal hypertension-related complications. In addition, we consider it important to monitor the portal venous pressure during the procedure to determine the correct end point.

In conclusion, IPSVS in a non-cirrhotic condition is rare. Here we present a case of a patient with hepatic encephalopathy due to congenital multiple IPSVS in both hepatic lobes, which were successfully treated by percutaneous transhepatic obliteration. Transcatheter embolization may be considered an effective therapy for patients who have symptomatic and refractory IPSVS. Caution is needed for portal hypertension-related complications after the embolization of IPSVS.

\section{Statement of Ethics}

There are no ethical conflicts to declare.

\section{Disclosure Statement}

The authors declare no conflicts of interest.

\section{References}

1 Pocha C, Maliakkal B: Spontaneous intrahepatic portal-systemic venous shunt in the adult: case report and review of the literature. Dig Dis Sci 2004;49:1201-1206.

$\checkmark 2$ Ohtomo K, Furui S, Saito M, Kokubo T, Itai Y, Iio M: Enormous intrahepatic communication between the portal vein and the hepatic vein. Clin Radiol 1986;37:513-514.

-3 Okada Y, Endo T, Kusano S, Yoshida M: Multiple intrahepatic portohepatic venous shunts: treatment with steel-coil embolization. AJR Am J Roentgenol 1991;157:971-973.

4 Maeda T, Mori H, Aikawa H, Komatsu E, Kagawa K: Therapeutic embolization of intrahepatic portosystemic shunts by retrograde transcaval catheterization. Cardiovasc Intervent Radiol 1993;16:245-247.

5 Yoshimitsu K, Andou H, Kudo S, Matsuo Y, Matsumoto S, Nakao T, Shimoda Y: Multiple intrahepatic portosystemic venous shunts: treatment by portal vein embolization. Cardiovasc Intervent Radiol 1993;16:49-51.

6 Hiraoka A, Kurose K, Hamada M, Azemoto N, Tokumoto Y, Hirooka M, Hasebe A, Kumagi T, Hirata M, Michitaka K, Minami H, Murakami M, Isobe Y, Horiike N, Onji M: Hepatic encephalopathy due to intrahepatic portosystemic venous shunt successfully treated by interventional radiology. Intern Med 2005;44:212-216.

7 Gupta V, Kalra N, Vyas S, Sodhi KS, Thapa BR, Khandelwal N: Embolization of congenital intrahepatic porto-systemic shunt by n-butyl cyanoacrylate. Indian J Pediatr 2009;76:1059-1060.

-8 Lee YJ, Shin BS, Lee IH, Ohm JY, Lee BS, Ahn M, Kim HJ: Intrahepatic portosystemic venous shunt: successful embolization using the Amplatzer Vascular Plug II. Korean J Radiol 2012;13:827-831.

-9 Yamagami T, Yoshimatsu R, Miura H, Hasebe T, Koide K: Hepatic encephalopathy due to intrahepatic portosystemic venous shunt successfully treated by balloon occluded retrograde transvenous embolization with GDCs. Acta Radiol Short Rep 2012;1:31.

10 Tanoue S, Kiyosue H, Komatsu E, Hori Y, Maeda T, Mori H: Symptomatic intrahepatic portosystemic venous shunt: embolization with an alternative approach. AJR Am J Roentgenol 2003;181:71-78.

-11 Kerlan RK, Jr., Sollenberger RD, Palubinskas AJ, Raskin NH, Callen PW, Ehrenfeld WK: Portal-systemic encephalopathy due to a congenital portocaval shunt. AJR Am J Roentgenol 1982;139:1013-1015.

12 Park JH, Cha SH, Han JK, Han MC: Intrahepatic portosystemic venous shunt. AJR Am J Roentgenol 1990;155:527-528. 


\section{Case Reports in Gastroenterology}
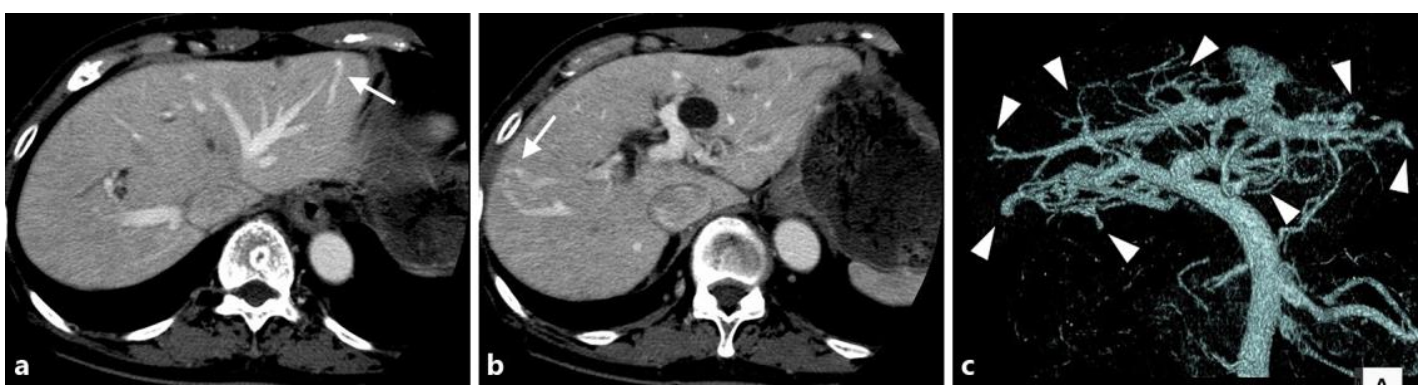

Fig. 1. Portal venous phase contrast-enhanced computed tomography (CT) images showing communications between the intrahepatic portal venous branch and hepatic veins in segment 5 (arrow in a) and segment 2 (arrow in b). A three-dimensional volume-rendering CT image showing multiple intrahepatic portosystemic venous shunts (arrowheads in c).

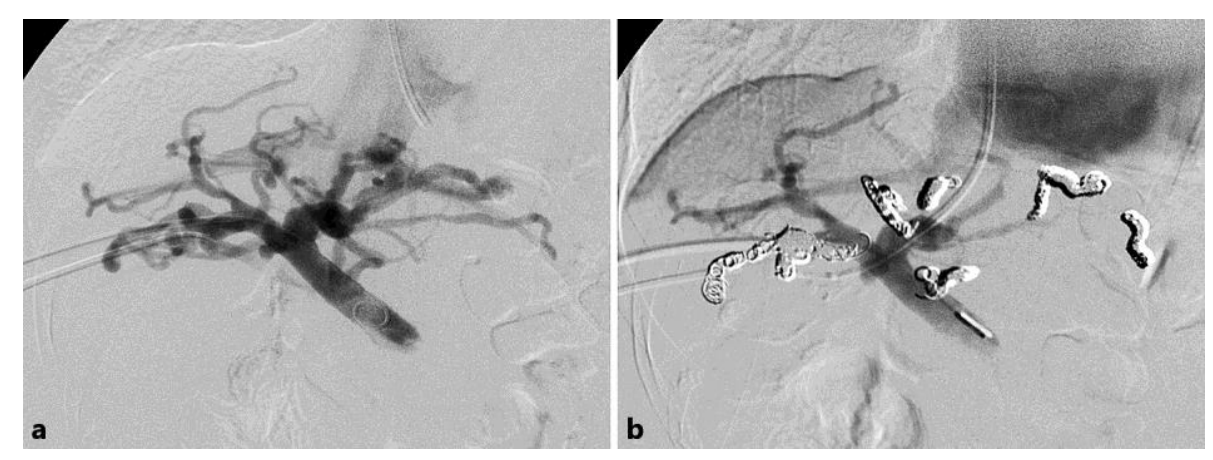

Fig. 2. a Percutaneous transhepatic portography showing multiple intrahepatic portosystemic shunts. b After embolization of the shunts by a large number of metallic coils, an obvious reduction in shunt flow was observed.

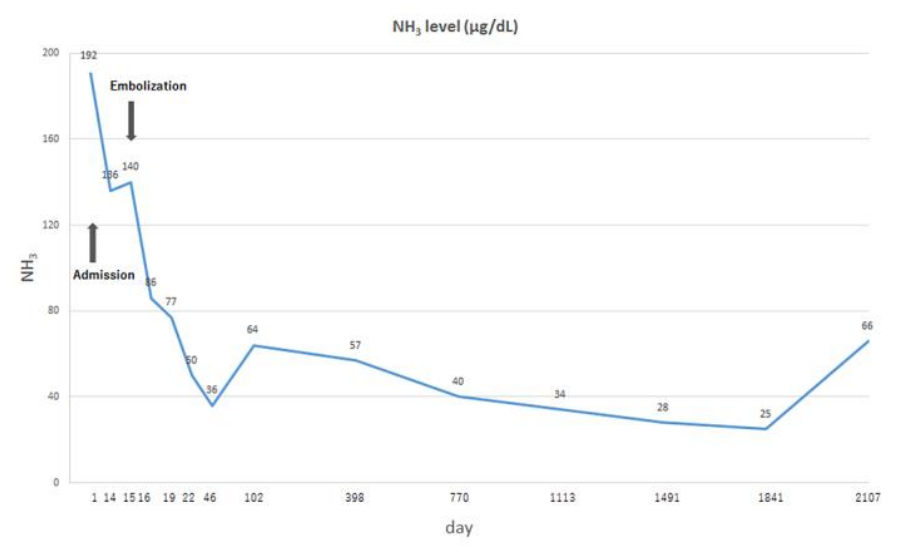

Fig. 3. Changes in serum ammonia $\left(\mathrm{NH}_{3}\right)$ level over time. On the day following the transcatheter embolization, serum ammonia level decreased to within normal range. In addition, normal serum ammonia levels were maintained for 5 years after embolization. 